\title{
THE $J$ FUNCTOR AND THE NONSTABLE HOMOTOPY GROUPS OF THE UNITARY GROUPS
}

\author{
MELVIN ROTHENBERG ${ }^{1}$
}

The recent work of Adams, Atiyah, Todd, and Walker on the complex Stiefel manifolds has certain implications regarding the structure of the nonstable homotopy groups $\pi_{i}\left(U_{m}\right), i>2 m$. The purpose of this note is to set up some of the machinery for investigating these implications. The information we have obtained so far concerning $\pi_{i}\left(U_{m}\right)$, $i>2 m$, is contained in Corollary I, stated below. It is likely that a more refined attack will yield better information. Theorems I and II are here regarded primarily as stepping stones to Corollary I; however, they may prove to be of some intrinsic interest.

The general situation we envisage is the following. Let $X$ be a finite C.W. complex and $f: S^{m} \rightarrow X$ a map. There are two cases.

1. The stable homotopy class of the map $f$ is of finite order. In this case, define co-degree $f=0$.

2. The stable homotopy class of the map $f$ is of infinite order, i.e., the induced map $H_{m}\left(S^{m} ; Q\right) \rightarrow H_{m}(X ; Q)$ is nontrivial. Then, by general theory, the map is $S$ rationally co-reducible. That is, there exists an integer $k$ and a map $h: S^{k}(X) \rightarrow S^{k+m}$ such that the composite $h s^{k}(f): S^{k+m} \rightarrow S^{k}(X) \rightarrow S^{k+m}$ is an isomorphism on $H_{m+k}\left(S^{k+m} ; Q\right)$. Given such an $S$ rational co-reduction, the map $h s^{k}(f): S^{k+m} \rightarrow S^{k+m}$ has a degree which we call, by abuse of notation, deg.(h). Among all $S$ co-reductions, there exists one of least positive degree $h^{\prime}$ (not necessarily unique) and the degree of any other $S$ co-reduction is a multiple of deg. $\left(h^{\prime}\right)$. Thus we can make the following definition:

co-degree $f=\operatorname{deg} .\left(h^{\prime}\right), h^{\prime}$ an $S$ rational co-reduction of minimal positive degree.

Among the spaces $X$ which fit into the above picture are the complex Grassmanians $V_{n+k, k}=U_{n+k} / U_{n}$. There is a standard map $S^{2 n+1}=U_{n+1} / U_{n} \rightarrow U_{n+k} / U_{n}$. The co-degree of this map will be denoted by $I_{k}^{n}$. We have

$$
I_{k}^{n} \neq 0, I_{1}^{n}=1 \text { and } I_{k}^{n}=0\left(\bmod I_{k-1}^{n}\right) .
$$

To state the properties of $V_{n+k, k}$ we need for our investigation, we now introduce a tool first exploited by Atiyah [4] and which lies be-

Presented to the Society, January 27, 1963; received by the editors November 12 , 1962.

1 This work was partially supported by NSF GP-623. 
hind the recent solution of Adams [1], Adams-Walker [2], AtiyahTodd [5] of some celebrated problems of algebraic topology. (See [3] for a detailed discussion.) We refer to the $J$ functor. For our purpose, a presentation due to Adams [3] is most convenient.

Let $F_{1}, F_{2}$ be two fiber spaces over $X$. A map $\mu_{1}: F_{1} \rightarrow F_{2}$ is called "fiberwise" if $\mu_{1}$ covers the identity map. $\mu_{1}$ is called a fiber homotopy equivalence if there exists a fiberwise map $\mu_{2}: F_{2} \rightarrow F_{1}$ such that $\mu_{1} \mu_{2}$ and $\mu_{2} \mu_{1}$ are homotopic to the identity via fiberwise maps.

Definition. Let $E_{1}, E_{2}$ be vector bundles over $X$ (real or complex). Then, $E_{1}, E_{2}$ are called $J$ equivalent

$$
E_{1} \tilde{J} E_{2},
$$

if the associated sphere bundles are fiber homotopically equivalent.

Now let $K_{\mathbf{\Delta}}(X)$ be the Grothendieck ring of stable vector bundles $(\Lambda=$ complex or real fields). We define a quotient group

$$
J_{\Lambda}(X)=K_{\Lambda}(X) / U_{\Lambda}(X),
$$

where $U_{\Lambda}(X)$ is the subgroup of elements of the form $\left[E_{1}\right]-\left[E_{2}\right]$, where $E_{1}, E_{2}$ are $J$ equivalent. $\left(U_{\Lambda}(X)\right.$ is a subgroup since

$$
E_{1} \tilde{J} E_{2}
$$

and

$$
\left.E_{1}^{\prime} \tilde{J} E_{2}^{\prime} \quad \text { implies } \quad E_{1} \oplus E_{1}^{\prime} \tilde{J} E_{2} \oplus E_{2}^{\prime} .\right)
$$

The decomposition

$$
K_{\Delta}(X)=K_{\Delta}(p)+\bar{K}_{\Lambda}(X)=Z+\bar{K}_{\Lambda}(X),
$$

where $p$ is a point and $\bar{K}_{\Delta}(X)$ is the reduced Grothendieck ring, induces a decomposition

$$
J_{\Lambda}(X)=J_{\Lambda}(p)+\bar{J}_{\Lambda}(X)=Z+\bar{J}_{\Lambda}(X) .
$$

Since every complex bundle can be considered a real bundle, and we can in turn complexify any real bundle, we have maps

$$
\lambda: J_{C}(X) \rightarrow J_{R}(X) \text { and } c: J_{R}(X) \rightarrow J_{C}(X) .
$$

We remark that $\lambda$ is a monomorphism. An important fact is that the groups $\bar{J}_{\Lambda}(X)$ are finite. The order of $x \in K_{R}(X)$ in $\bar{J}_{R}(X)$ is what is usually called the stable $J$ order of $x$.

To study the co-index of the complex Stiefel manifolds, one looks at the groups $\bar{J}_{C}\left(C P_{k}\right)$, where $C P_{k}$ is the complex projective space of $k-1$ complex dimensions. Specifically, Atiyah has shown that $I_{k}^{n}=1$ iff $n L_{k}$ is $\bar{J}$ equivalent to 0 , where $L_{k}=$ canonical complex line bundle over 
$C P_{k}$, that is, iff $n=0\left(\bmod m_{k}\right)$, where $m_{k}$ is the order of $L_{k}$ in $\bar{J}_{C}\left(C P_{k}\right)$ [4]. Using this fact, he and the others mentioned have computed those pairs $(n, k)$ for which $I_{k}^{n}=1[2 ; 5]$. One might suspect that, in general, $I_{k}^{n}$ is closely related to the stable $\bar{J}$ order of $n L_{k}$. This is, in fact, true, as the following theorem shows.

First let us define new integers $m_{\boldsymbol{k}}^{n}$.

Definition. Let $m_{\mathbf{k}}^{n}$ be the order of $n$ in $Z / m_{k} Z$.

Notice $m_{k}^{1}$ is just $m_{k}$ and in general $m_{k}^{n}$ is the order of $n L_{k}$ in $\bar{J}_{C}\left(C P_{k}\right)$. These integers are much more convenient than $I_{k}^{n}$ since they are computable. They are related as follows.

THEOREM I. $I_{\boldsymbol{k}}^{n}$ and $m_{\mathbf{k}}^{n}$ have the same prime factors.

REMARK. That $\left(I_{k}^{n}\right)^{p}=0\left(\bmod m_{k}^{n}\right)$ is due essentially to Adams [3]. One might conjecture $I_{k}^{n}=m_{k}^{n}$, but we are unable to prove it.

A relation between the general situation and our model, the complex Grassmanians, is given in the following theorem.

TheOREM II. Let $f: S^{2 n+1} \rightarrow X$ be a map such that:

1. $0<$ co-degree $f$;

2. the prime divisors of the co-degree $f$ are $\leqq n$;

3. $\operatorname{dim} X \leqq 2(n+k)$;

4. there exists a complex $n$ plane bundle over $X, E$, such that it is $S$ trivial and such that $f^{*}(E)=E^{\prime}$, the standard complex reduction of the tangent bundle of $S^{2 n+1}$.

Then, $\left(m_{\mathbf{k}}^{n}\right)^{q}=0(\bmod$ co-degree $f)$ for some integer $q$.

REMARKs. Hypothesis 2 is not overly restrictive. In fact, we have:

Proposition I. Suppose $\operatorname{dim} X<4 n+1$ and $f: S^{2 n+1} \rightarrow X_{2 n+1}$ (the $2 n+1$ skeleton of $X)$ is co-reducible. Then the prime divisors of the codegree of $f$ are $\leqq n$.

Sketch OF PROOF. The $p$ primary component of the stable homotopy group, $\pi_{m}=\pi_{m+n}\left(S^{n}\right), n$ sufficiently large, is 0 if $m<2 p-3$. (See $\mathrm{Hu}[6$, Chapter XI].) Now, a simple induction argument constructing $X$ one cell at a time from its $2 n+1$ skeleton yields the result.

As is well known, a complex $n$ plane bundle is $S$ trivial on $X$ if its Chern classes vanish and $H^{*}(X ; Z)$ has no torsion. Thus, in practice, the condition in Hypothesis 4 that $E$ be $S$ trivial is not too difficult to check.

The fewer prime divisors of $m_{k}^{n}$, the more information the theorem yields. In particular, if $m_{k}^{n}=1$, the theorem asserts the map $f$ has a right $S$ homotopy inverse.

We now use the above theorem to gain information about the groups $\pi_{i}\left(U_{m}\right)$. 
Corollary I. Consider the standard fibration, $S^{2 n+1} \rightarrow B U_{n} \rightarrow B U_{n+1}$. Let $1<r<2 k \leqq 2 n$. Then, the kernel $\pi_{2 n+r}\left(S^{2 n+1}\right) \rightarrow \pi_{2 n+r}\left(B U_{n}\right)$ consists only of classes $x \in \pi_{2 n+r}\left(S^{2 n+1}\right)$ such that $\left(m_{k}^{n}\right)^{q}=0(\bmod$ order $x)$ for some $q$.

Proof. Let $M=E_{2 n+r+1} \cup_{x} S^{2 n+1}$. Let $\mu$ be the map, $\mu: S^{2 n+1} \rightarrow B U_{n}$. Then the map $\mu$ can be extended to $\mu^{\prime}: M \rightarrow B U_{n}$ iff $x$ is in the kernel of $\pi(\mu)$. The map $\mu$ can be extended iff the map $\iota: S^{2 n+1} \rightarrow M$ satisfies the hypothesis of Theorem II. Thus $\left(m_{k}^{n}\right)^{q}=0(\bmod$ co-degree $\iota)$. But the co-degree $\iota=$ the order of $x$ in $\pi_{2 n+r}\left(S^{2 n+1}\right)$. Q.E.D.

Corollary I along with the previous remarks imply:

Corollary II. Suppose $n=0\left(\bmod m_{k}\right)$. Then, $\pi(\mu): \pi_{2 n+r}\left(S^{2 n+1}\right)$ $\rightarrow \pi_{2 n+r}\left(B U_{n}\right), 1<r \leqq 2 k$, is a monomorphism.

The formula for $m_{k}$ is as follows [3]: For any prime $p$, let $\gamma_{p}(a)$ be the exponent of $p$ occurring in the prime factorization of $a$. Then

where

$$
\begin{aligned}
& \gamma_{p}\left(m_{k}\right)=0 \quad \text { if } p>k \\
& \gamma_{p}\left(m_{k}\right)=\operatorname{Sup}_{r}\left(r+\gamma_{p}(r)\right) \quad \text { if } p \leqq k,
\end{aligned}
$$

$$
1 \leqq r \leqq k-1 / p-1
$$

Proof of Theorem I. The proof of Theorem I splits into two parts. It suffices to prove the two statements:

a. $\left(I_{\mathbf{k}}^{n}\right)^{p}=0\left(\bmod m_{\mathbf{k}}^{n}\right)$;

b. $\left(m_{k}^{n}\right)^{q}=0\left(\bmod I_{k}^{n}\right)$.

The proof proceeds via a well-known reduction. If $E$ is a vector bundle over a space $X$, we denote by $X^{E}$ the Thom space of $E$. We are particularly interested in $X=C P_{k}$ and $L$ the canonical complex line bundle. Now $C P_{k}^{n L}$ is an $S$ retract of $V_{n+k, k}[4 ; 7]$. Thus, $I_{k}^{n}$ is also the co-index of the canonical map $S^{2 n} \rightarrow C P_{k}^{n L}$. For the moment, we simply ignore the complex structure and consider all bundles as real bundles. Let $R$ denote the trivial (real) bundle of real dimension $R$. Then we have a map for some $R, \mu: C P_{\mathbf{k}}^{n L+R} \rightarrow S^{n L+R}$ such that its composition with the canonical map $S^{2 n+R} \rightarrow C P_{k}^{n L+R}$ is of degree $I_{\mathbf{k}}^{n}$.

The proof of (a) proceeds as follows. Let $S(n L+R+1)$ denote the associated sphere bundle to $n L+R+1$ over $C P_{k}$. Then we have a map $\lambda: S(n L+R+1) \rightarrow C P_{\mathbf{k}}^{n L+R}$ which is a homeomorphism when restricted to each of the fibers. This induces a map $\lambda^{\prime}: S(n L+R+1) \rightarrow S^{2 n+R}$ which is a map of degree $I_{k}^{n}$ on each of the fibers. If $\pi$ is the map $S(n L+R+1) \rightarrow C P_{k}$, then the map $\lambda^{\prime} \times \pi: S(n L+R+1) \rightarrow S^{2 n+R}$ $\times C P_{k}$ is a fiberwise map of degree $I_{k}^{n}$ on each of the fibers. According to Theorem 4 of Adams [3], the stable $J$ order of $(n L+R+1)$ is a 
divisor of $\left(I_{k}^{n}\right)^{p}$. But the stable $J$ order of $(n L+R+1)$ is the stable $J$ order of $n L$, which is exactly $m_{k}^{n}$. Thus $\left(I_{k}^{n}\right)^{p}=0\left(\bmod m_{k}^{n}\right)$. Q.E.D.

The proof of (b) will take a little longer, since we do not have Adams to help us. The first step involves examining the structure of $K_{C}\left(C P_{k}\right)$. The proof of the following can be found in [1]. In any case, it is not difficult. $K_{C}\left(C P_{k}\right)=Z[x] /\left[x^{k}\right]$ as an algebra over $Z$. The generator $x$ can be chosen so that $1+x$ is represented by the canonical line bundle $L$. To obtain the properties we want, we have to study the homomorphism $J: K_{C}\left(C P_{k}\right) \rightarrow J_{C}\left(C P_{k}\right)$ on $x^{r}$ and $L^{r}$. In general, $J$ does not behave well with respect to multiplication; however, the complex projective spaces have special properties which we can utilize.

$C P_{k}$ can be regarded as the $2 k-2$ skeleton of $C P_{\infty}$ which is a $K(Z, 2)$ space and, in particular, a topological group which is a $Z$ module. Thus for each integer $r$, there is a map $r: C P_{\infty} \rightarrow C P_{\infty}$; hence, by using cellular approximation if necessary, a map $r: C P_{k} \rightarrow C P_{k}$. It is easily checked, using the Chern character, that $r^{*}(L)=L^{r}$. Thus we have

LEMmA I. If $m J(x)=0$, then $m J\left(x^{r}\right)=0$ for each $r>0$.

Proof. By induction on $r . J(m x)=0$ implies $J\left(r^{*}(m x)\right)=0$ implies $J\left(r^{*}(m(L-1))\right)=0$ implies $J\left(m r^{*}(L-1)\right)=0$ implies $J\left(m\left(L^{r}-1\right)\right)=0$ implies $J\left(m\left((x+1)^{r}-1\right)\right)=0$. Now expanding $(x+1)^{r}$ and using the induction hypothesis, we get the result.

Lemma II. Suppose $m q=0\left(\bmod m_{k}\right)$. Then there exists a p such that $\bar{J}\left(m L^{a}\right)=0\left(\right.$ in $\left.\bar{J}_{C}\left(C P_{k}\right)\right)$, where $a=q^{p}$.

Proof. If $q=1$, then Lemma I says any $p \neq 0$ will do. Otherwise, we can choose $p$ so large that

1. $x^{a}=0$;

2. $(x+1)^{a}=x^{a}+1(\bmod q)=1(\bmod q)$.

In fact, choosing $p=k$ and using the fact that $x^{k+1}=0,(2)$ is easily shown to be satisfied. Now $J\left(m L^{a}\right)=m J\left((x+1)^{a}\right)$. By $(2),(x+1)^{a}$ is of the form $1+q\left(\sum_{j} b_{j} x^{j}\right)$. Now $J\left(m\left(1+q\left(\sum_{j} b_{j} x^{j}\right)\right)\right)=J(m)$ $+J\left(m q\left(\sum_{j} b_{j} x^{j}\right)\right)$. The second term is 0 by the hypothesis and Lemma I. Thus $J\left(m L^{a}\right)=J(m)$. Hence $\bar{J}\left(m L^{a}\right)=0$. Q.E.D.

So far we have used the existence of a map $L^{r} \rightarrow L$ covering a map $C P_{k} \rightarrow C P_{k}$. However, for a line bundle there is a map going the other way also.

LEMma III. Let $L$ be a complex line bundle over a space $X$. Then for each $r$, there exists a fiberwise map $\mu: L \rightarrow L^{r}$. Futher, inner products can be chosen so that $\mu$ preserves norms. Finally, the induced map on the associated circle bundles has degree $r$ on each of the fibers. 
Proof. Let $L^{\prime}$ be the associated circle bundle to $L$. Then $L^{\prime}$ is a principal bundle with group $S$, the circle. The representation $r: S \rightarrow S$ of $e^{i \theta} \rightarrow e^{i r \theta}$ induces another principal $S$ bundle over $X, L^{\prime \prime}$. By definition, the associated line bundle is just $L^{r}$. Now

$$
L^{\prime \prime}=S \underset{S}{\times} L^{\prime},
$$

where the action of $S$ on itself is via the representation $r$. Thus the map $L^{\prime} \rightarrow L^{\prime \prime}$ taking $e \rightarrow(1 \times e)$ is a fiberwise map which is of degree $r$ on each of the fibers. The map $r: S \rightarrow S$ extends to a norm preserving map $C \rightarrow C, q e^{i \theta} \rightarrow q e^{i r \theta}$. Thus the fiberwise map extends to one $\mu^{\prime}: C \times{ }_{S} L^{\prime} \rightarrow C \times{ }_{s} L^{\prime \prime}$. Putting the Hermitian inner product on $C \times{ }_{S} L^{\prime}$ (respectively $C \times{ }_{S} L^{\prime \prime}$ ) induced from $C \times L^{\prime}$ (respectively $\left.C \times L^{\prime \prime}\right)$, we see that $\mu^{\prime}$ is norm preserving. Now $L=C \times{ }_{S} L^{\prime}$ and $L^{r}=C \times{ }_{S} L^{\prime \prime}$ (by definition), and the lemma follows.

It is obvious from the definition that we have

COROLlaRY III. The fiberwise map $L \rightarrow L^{r}$ induces a fiberwise norm preserving map $m L \rightarrow m L^{r}$ of degree $r^{m}$ on the fibers of the associated sphere bundles.

Now by the same argument used in the proof of (a) and by Corollary III, we have Corollary IV.

CorollaRy IV. There exists a map $C P^{m L} \rightarrow C P^{m E}$, where $E=L^{r}$, such that the composite map $S^{2 m} \rightarrow C P^{m L} \rightarrow C P^{m E}$ is homotopic to $r^{m}$ times the canonical map $S^{2 m} \rightarrow C P^{m E}$.

We can now complete the proof of (b). Letting $q=m_{\mathbf{k}}^{n}$ in Lemma II, we have $\bar{J}\left(n L^{a}\right)=0$, where $a=q^{p}$ for sufficiently large $p$. Using $a$ for $r$ in Corollary IV and the fact that $C P^{n E}$, where $E=L^{a}$, is $S$ co-reducible, it follows that the co-index of $S^{2 n} \rightarrow C P^{n L}$ divides $a^{n}=q^{t}$ for some $t$. Thus $\left(m_{k}^{n}\right)^{t}=0\left(\bmod I_{k}^{n}\right)$. Q.E.D.

Proof of Theorem II. We consider the fibration $U_{n} \rightarrow U_{n+k}$ $\rightarrow V_{n+k, k}$. We also have the fibration $V_{n+k, k} \rightarrow B U_{n} \rightarrow B U_{n+k}$. For any finite C.W. complex $X$ and any map $f: S^{2 n+1} \rightarrow X$, we have a ladder of exact sequences:

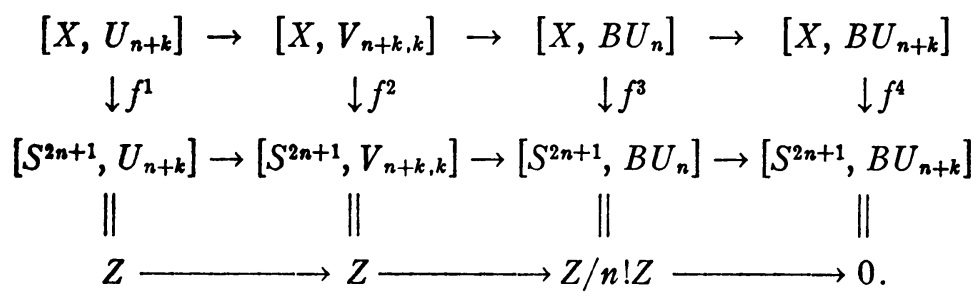


Now let us apply the hypothesis of Theorem II. Hypothesis 4 implies there is a $\mu^{\prime} \in\left[X, B U_{n}\right]$ such that $f^{3}\left(\mu^{\prime}\right)=i$, a generator of $Z / n ! Z$. Hypotheses 3 and 4 imply there is such a $\mu^{\prime}$ which goes to 0 in $\left[X, B U_{n+k}\right]$. This $\mu^{\prime}$ pulls back to $\mu, \mu \in\left[X, V_{n+k, k}\right]$ such that $f^{2}(\mu)$ $=1(\bmod n !)$. Thus the composite $\mu f: S^{2 n+1} \rightarrow X \rightarrow V_{n+k, k}$ satisfies $\mu f=1(\bmod n !)$, where $\mu f \in \pi_{2 n+1}\left(V_{n+k, k}\right)=Z$. Now, passing to the stable case, we have a co-reduction $t: S^{r}\left(V_{n+k, k}\right) \rightarrow S^{2 n+r+1}$. Recalling the definition of $I_{k}^{n}$, we can choose $t$ such that $t s^{r}(\mu) s^{r}(f)=I_{k}^{n}(\bmod n !)$ as elements of $\pi_{2 n+r+1}\left(S^{2 n+r+1}\right)=Z$. Let $g=t s^{r}(\mu) s^{r}(f)$. Choose an integer $p$ so that $(n !)^{p}=0(\bmod \operatorname{co-index} f)$. Then $\left(g-I_{k}^{n}\right)^{p}=0\left(\bmod n !^{p}\right)$. Thus, $m g=\left(I_{k}^{n}\right)^{p}\left(\bmod n !^{p}\right)$ for some $m$. Since $(n !)^{p}=0(\bmod c o-i n d e x$ $f)$, we have $m g=\left(I_{q}^{n}\right)^{p}(\bmod \operatorname{co}-\operatorname{ind} \operatorname{dex} f)$. But $m g$ is a co-reduction of $f$. Thus $m g=0(\bmod c o-i n d e x f)$. Therefore, $\left(I_{k}^{n}\right)^{p}=0(\bmod \operatorname{co}-i n d e x f)$. Now applying Theorem I yields Theorem II. Q.E.D.

Final REMARK. The methods of proof in this note are highly nongeometric, not because of choice but because we could not prove them any other way. Geometrically the situation should be viewed as follows. There is a sequence of sphere bundles

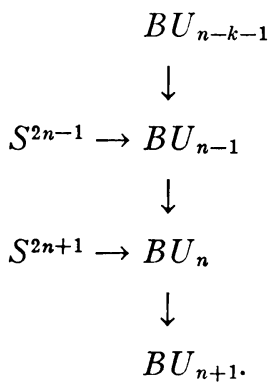

Let $\mu_{i}: S^{2 i+1} \rightarrow B U_{i}$ be the maps in the fiber spaces above. Suppose for simplicity we consider the case $m_{k}^{n}=1$. Then, Corollary II says $\pi\left(\mu_{n}\right): \pi_{2 n+r}\left(S^{2 n+1}\right) \rightarrow \pi_{2 n+r}\left(B U_{n}\right), 1<r<2 k$ is a monomorphism. However, one also knows that $m_{k}^{n}=1$ iff $\mu_{n-1}$ factors back through a map $\mu_{n-1}^{\prime}: S^{2 n-1} \rightarrow B U_{n-k-1}$. That is, if the complex tangent bundle on $S^{2 n-1}$ admits a nonzero $k$ frame. Thus one geometric statement implies another. It would be more satisfactory if one could see this relationship via geometric constructions. One might hope that there is some general duality involved which has stronger implications.

\section{BIBLIOGRAPHY}

1. J. F. Adams, Vector fields on spheres, Ann. of Math. (2) 75 (1962), 603-632.

2. J. F. Adams and G. Walker, On complex Stiefel manifolds, (to appear).

3. J. F. Adams, Applications of the Grothendieck-Atiyah-Hirzebruch functor $K(X)$, 
Colloq. Algebraic Topology, 1962, pp. 104-113, Matematisk Institut, Aarhus Universitet, Denmark. 310.

4. M. F. Atiyah, Thom complexes, Proc. London Math. Soc. (3) 11 (1961), 291-

5. M. F. Atiyah and J. A. Todd, On complex Stiefel manifolds, Proc. Cambridge Philos. Soc. 56 (1960), 342-353.

6. Sze-Tsen Hu, Homotopy theory, Pure and Applied Mathematics VIII, Academic Press, New York and London, 1959.

7. I. M. James, Spaces associated with Stiefel manifolds, Proc. London Math. Soc. (3) 9 (1959), 115-140.

University of Chicago

\section{CURVATURE IN NILPOTENT LIE GROUPS}

JOSEPH A. WOLF

1. Introduction. The purpose of this note is to prove:

TheOREM. Let $M$ be a Riemannian manifold which admits a transitive connected noncommutative nilpotent Lie group of isometries. Given $x \in M$, there exist 2-dimensional subspaces $R, S$ and $T$ of the tangentspace $M_{x}$ such that sectional curvatures satisfy

$$
K(S)<0<K(T) \text { and } K(R)=0 .
$$

Corollary. Let $G$ be a connected nilpotent Lie group, let $B$ be a positive definite symmetric bilinear form on the Lie algebra (\$) of $G$, and let $M$ be the Riemannian manifold obtained by left translation of $B$ to every tangentspace of $G$. Then these are equivalent:

1. $M$ has a positive sectional curvature.

2. $M$ has a negative sectional curvature.

3. $G$ is not commutative.

To prove the Corollary from the Theorem, one simply observes that $M$ must be flat if $G$ is commutative.

The interest of the Theorem and its Corollary is based on the deep similarity between nilpotent Lie groups and Riemannian manifolds of nonpositive sectional curvature. Two striking points of similarity are their coverings (compare [4] with $\$ 4.2$ of [3]) and their exponential mappings. The results of this note show that the class of Riemannian manifolds obtained by placing left invariant metrics on nilpotent Lie groups is quite different from the class of Riemannian manifolds of nonpositive sectional curvature. In the nonflat case one

Received by the editors November 27, 1962. 